\title{
A Hidden Pattern Discovery and Meta-synthesis of Preference Adjustment in Group Decision-Making ${ }^{*}$
}

\author{
Huizhang Shen ${ }^{1}$, Jidi Zhao ${ }^{1}$, and Huanchen Wang ${ }^{2}$ \\ ${ }^{1}$ Department of Information Systems, Shanghai Jiao Tong University, 535 Fahuazhen Rd., \\ Shanghai, China 200052 \\ \{Hzshen, Judyzhao33\} asjtu . edu.cn \\ ${ }^{2}$ Institute of System Engineering, Shanghai Jiao Tong University, 535 Fahuazhen Rd., \\ Shanghai, China 200052
}

\begin{abstract}
Two aspects of group decision-making (GDM) have received much attention since its appearance, one is the organizing process of GDM which emphasizes in particular on behavioral science and qualitative analysis, and the other is the weight allocation and meta-synthesis of group preference focusing on the quantificational computation. Despite the abundant researches, the existing solutions do not take into account the dynamic change of group members' decision-making preference in the GDM process. This paper considers the preference change in GDM as a dynamical procedure, researches into the important hidden pattern in GDM process, and puts forward a GDM meta-synthesis method based on Markov Chain to mine the hidden information with both qualitative analysis and quantificational computations.
\end{abstract}

Keywords: group decision-making, hidden pattern, meta-synthesis.

\section{Introduction}

Based on the construction of group preference, group decision-making is a procedure [1] of synthesizing the preferences of each decision-maker in the group and sorting the schemes or choosing the best scheme in the scheme set. One of the key points in group decision-making is the decision-makers' preference relations. As Arrow pointed, the preference relation formed by group decision should satisfy five rational terms [1] which are the preference axiom, impossible axiom, completeness, Pareto optimization, and non-autarchy. Notable scholars such as Arrow [1], Dyer [2], Keeney [3] and French [4] established the theoretical foundation about group decision preference relation analysis in the group decision-making research. From their research, we know that the group preference is a function of individual preferences in the issue of the group decision-making. Preference is a term quoted from economics. In the group decision-making problems, it is used to represent the decision-makers' partiality on value [2]. There has been much research on the meta-synthetic problem. The metasynthetic algorithms accepted widely include Weighted Average Method, Bordly Multiplication [5], Bayesian Integration Method [3], Entropy Method [4],

* This research is supported by NSFC(70671066). 
Fuzzy Cluster Analysis [2], and so on. The dynamics of the group aggregation of individual decisions has also been a subject of central importance in decision theory [6]. In order to narrow the gap between the collective opinion and each decision maker's opinion, Fan et al [8] proposed a linear goal programming model to solve group decision-making (GDM) problems where the preference information on alternatives provided by decision makers is represented in two different formats, i.e. multiplicative preference relations and fuzzy preference relations. Hochbaum and Levin [6] present a model to quantify the degree of preference which allows for flexibility in decision protocols and can take into consideration imprecise beliefs. Based on fuzzy linguistic preference relations, Herrera-Viedma et al [8] present a model of consensus support system to assist the experts in the consensus reaching process of group decision-making problems.

Despite the abundant research on group decision-making problems, existing solutions do not consider the preference adjustment as a continuous procedure and ignore the effect of the dynamic change procedure of group members' decision-making preference on group decision-making process. The procedure of forming individual preference is a decision-maker's meta-synthetic thinking procedure of perceiving all the information relating to expectation, information, sensibility, creativity, and so on, thus it is a extremely complex procedure [5]. Generally speaking, the decision-making group's preference on the scheme set will change as the decision-makers adjust their preferences under the same decision-making rules. We call it the preference convergence procedure which can be described as follows. Suppose that every member of the decision-making group has the will of coming to a unified decision. Each decisionmaker in the group $G$ presents her own preference based on the utility maximization rules. The $r^{\text {th }}$ decision-maker $D M_{r}$ will adjust and readjust her reference and feed it back to the group of decision-makers after he cognizes the preferences of the other $(l-1)$ decision-makers. This procedure of cognizing and feedback goes with each member in the group repeatedly and continuously. Such a procedure usually repeats finite times, for example, there are usually five rounds in Delphi. In the group decisionmaking procedure, communication among decision-makers is encouraged to increase their comprehension on the decision-making problem, on the available information and even on themselves. The decision makers are also encouraged to continuously adjust their preferences until they come to consensus on the group decision-making.

Thus in this paper, we focus on the research of how to achieve consensus quickly and reliably in group decision-making process. We consider the preference change in GDM as a dynamical procedure, research into the important hidden pattern in GDM process, study the meta-synthetic procedure of group preference based on the decision-makers' preference utility value, and put forward a meta-synthesis approach based on Markov chain for group decision-making problems.

\section{Group Decision-Making and A Hidden Pattern of Group Preference Adjustment}

In traditional meta-synthetic approaches on group decision-making, once the weights for every decision-maker are fixed on, the group preference value on each scheme is 
determined by the sum of each decision-maker's weight multiplied by her current preference value of the scheme. Then all schemes are sorted in the order of their preference values. For example, if the order is $x^{2} R x^{1} R x^{3} R x^{4}$, the scheme $x^{2}$ will be the group's preferred scheme. Nevertheless, here a decision-maker's current preference value vector in the scheme set is a transient decision instead of a stationary one. That is, the decision-maker may have changed her preference value from a value into the current one in this round, and she may continue to change her preference value in the rounds hereafter. Therefore, in traditional meta-synthetic approaches, ignoring the dynamic procedure of decision-maker's preference adjustment and decision-maker's hereafter current preference values may lose important information in group decisionmaking. As the group decision-making procedure continues, the current preference values are supposed to change along the time. Thus we consider the dynamic procedure of decision-maker's preference adjustment and its effect on group decisionmaking in this paper.

\subsection{Discrete Time Markov Chains}

A sequence of random variables $\left\{E_{n}\right\}$ is called a Markov chain if it has the Markov property:

$$
\begin{aligned}
& T\left\{E_{n+1}=j \mid E_{n}=i, E_{n-1}=i_{n-1}, \ldots, E_{0}=i_{0}\right\}=T\left\{E_{n+1}=j \mid E_{n}=i\right\} \\
& T_{i j}=T\left\{E_{n+1}=j \mid E_{n}=i\right\}
\end{aligned}
$$

Here, $E_{i}$ is an event and $T_{i j}$ is the probability to transit from state $i$ to state $j$ of the event. The property is called Memoryless. In other words, "Future" is independent of "Past" given "Present". Here the transition probabilities $T_{i j}$ satisfy $T_{i j} \geq 0, \quad \sum_{j=0}^{\infty} T_{i j}=1$.

The Chapman-Kolmogorov equation for a discrete-time Markov chain is as follows, if the distribution at "time" $t_{n}$ is $\pi^{(n)}$, then the distribution at "time" $t_{n+1}$ is given by

$$
\pi^{(n+1)}=\pi^{(n)} T
$$

\subsection{Hidden Pattern of Group Preference Change Based on Markov Chain and the Construct of Markov State Transition Matrix for GDM}

After the $t$ rounds adjustment, the preference utility values in all the rounds for decision-maker $D M_{r}$ are 


$$
\pi_{r}=\left\{\begin{array}{cccc}
\pi_{r}^{1}\left(x^{1}\right) & \pi_{r}^{1}\left(x^{2}\right) & \cdots & \pi_{r}^{1}\left(x^{s}\right) \\
\pi_{r}^{2}\left(x^{1}\right) & \pi_{r}^{2}\left(x^{2}\right) & \cdots & \pi_{r}^{2}\left(x^{s}\right) \\
\cdots & \cdots & \cdots & \cdots \\
\pi_{r}^{t}\left(x^{1}\right) & \pi_{r}^{t}\left(x^{2}\right) & \cdots & \pi_{r}^{t}\left(x^{s}\right)
\end{array}\right\}
$$

In this matrix, each row stands for the preference utility value vector in each round. Comparing the $k^{\text {th }}$ row with the $(k+1)^{\text {th }}$ row $(\{k=1,2, \cdots, t\})$, if there exists $\pi_{r}^{k+1}\left(x^{i}\right) \downarrow \Leftrightarrow \pi_{r}^{k+1}\left(x^{j}\right) \uparrow$, we set the state variable $E_{i j}=E_{i j}+1$, which shows that the decision-maker has ever changed her preference from the scheme $x^{i}$ to $x^{j}$. For each decision-maker, there are at most $t-1$ times of adjustment. Packing all the adjustment for the group together, we have

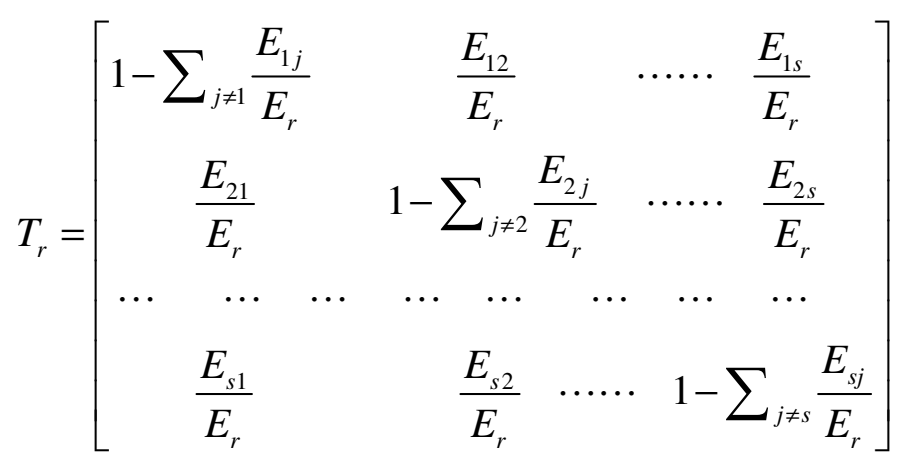

where $T_{r}$ is the preference state transition matrix for decision-maker $D M_{r}, E_{i j}$ denotes the preference transition times from $x^{i}$ to $x^{j}$ and $E_{r}=t-1$ is the sample space for the state transition times.

For example, the preference utility value matrix for decision-maker $D M_{r}$ is

$$
\Lambda_{r}=\left[\begin{array}{llll}
0.1 & 0.3 & 0.2 & 0.4 \\
0.2 & 0.2 & 0.3 & 0.3 \\
0.2 & 0.3 & 0.2 & 0.3 \\
0.2 & 0.4 & 0.2 & 0.2 \\
0.3 & 0.3 & 0.2 & 0.2
\end{array}\right]
$$

The first row of the matrix is the initial value and the sample space is $t-1=5-1=4$.

Comparing the second row with the first row, we have $x^{2} \rightarrow x^{1}, x^{4} \rightarrow x^{3}$.

Comparing the third row with the second, we have $x^{3} \rightarrow x^{2}$.

Comparing the fourth row with the third, we have $x^{4} \rightarrow x^{2}$. 
And, comparing the fifth row with the fourth, we have $x^{2} \rightarrow x^{1}$.

According to equation (4), we have the preference state transition matrix $T_{r}$ for decision-maker $D M_{r}$ is

$$
T_{r}=\left[\begin{array}{cccc}
1 & 0 & 0 & 0 \\
0.5 & 0.5 & 0 & 0 \\
0 & 0.25 & 0.75 & 0 \\
0 & 0.25 & 0.25 & 0.5
\end{array}\right]
$$

In this matrix, $\frac{E_{11}}{E_{r}}=1$ shows that the decision-maker never changes her preference on $x^{1}$.

Define the overall state transition matrix of the decision-making group in the $t$ rounds adjustment procedure as

$$
T=\frac{1}{l} \sum_{r=1}^{l} T_{r}
$$

\subsection{Markov Property of the Group Preference Adjustment Procedure}

Because each decision-maker in the group independently puts forward her preference judgment matrix, the preference state $E_{n}^{r}$ of decision-maker $D M_{r}$ is independent of other decision-makers and the future preference state $E_{n+1}^{r}$ is independent of other states except the current state $E_{n}^{r}$, thus the group decision-making procedure satisfies equation (1).

Obviously, the transition probabilities $T_{i j}$ constructed from equation (4) satisfy $T_{i j} \geq 0, \quad \sum_{j=0}^{\infty} T_{i j}=1$.

Equation (5) shows that the overall state transition probabilities matrix is the mean value of the matrices of transition probabilities of each decision-maker, thus the group property is implied in the individual properties.

Therefore, we can use the Chapman-Kolmogorov equations, equation (2), to get $\pi^{(n+1)}$ at "time" $t_{n+1}$ from $\pi^{(n)}$ at "time" $t_{n}$.

\section{Implementation of Markov-Based Meta-synthesis Approach for Group Decision-Making}

A weight allocation method in group decision-making, based merely on the analysis of the objective individual preferences in the scheme set without man-made subjective 
factors and taking into account both the individuals' carefulness and extremeness in the group, is presented in [9]. Shen et al [10] presented a clustering algorithm of group decision-making preference. The weight allocation method and the clustering algorithm will be introduced into the group decision-making meta-synthesis approach, which is based on Markov chain and works as follows.

(1) Publish the group decision-making problem and its background to each group member, including the event, the available data and information, the constraints, the schemes, the decision-making rules, the user handbook for GDSS, etc.

(2) Each decision-maker gives her preference judgments between each two schemes in the scheme set. All the decision-makers can publish their opinions, evidences and explanations on the message board to support their points of view.

(3) Based on the preference judgment matrix, the GDSS automatically generates the preference utility values for a decision-maker $D M_{r}$ in the $t^{\text {th }}$ round, $\left\{\pi_{r}^{t}\left(x^{1}\right), \pi_{r}^{t}\left(x^{2}\right), \cdots, \pi_{r}^{t}\left(x^{s}\right)\right\}$. As stated above, the individual preference adjustment in group decision-making is a continuous procedure in which the decisionmakers adjust their preference in each round respectively based on the communications among the group members. The continuous adjustments make the group decisions come to converge gradually. The preference utility values vector $\left\{\pi_{r}^{t}\left(x^{1}\right), \pi_{r}^{t}\left(x^{2}\right), \cdots, \pi_{r}^{t}\left(x^{s}\right)\right\}$ for each decision-maker is preserved for constructing the Markov state transition matrix in step (8).

(4) Packing all the preference values got in step (3), the system has the preference utility values matrix for the $t^{\text {th }}$ round, automatically computes the preference distance $d_{i j}$ between decision-maker $D M_{i}$ and $D M_{j}$ on the scheme set, constructs the preference difference matrix $D$, clusters the preference difference matrix $D$ using the algorithm given in [10] and displays the clustering results on the message board.

(5) The decision-makers in the group adjust their preferences using the clustering information given by the system. Go back to step (2) and begin another round of preference judgment.

(6) Repeat through step (2) to step (5) for $t=7 \pm 2$ times. The choice of $\mathrm{t}=7 \pm 2$ is based on two foundations, one is that conventional Delphi method usually repeats more than four times, the other is psychological research shows that $7 \pm 2$ is an experiential value for human being's thought span. The specific value for $\mathrm{t}$ is given by the organizer according to the time limitation and the rigor of the problem before the beginning of group decision-making.

(7) Based merely on the decision-makers' preference judgment information, the system calculates the weight assigned to each decision-maker and yields the weight vector $W=\left(w_{1}, w_{2}, \cdots, w_{s}\right)$ for the group using the weight allocation method given in [9]. 
(8) Construct the Markov state transition matrix $T$ using equation (4) and (5) with the saved preference values $\left\{\pi_{r}^{t}\left(x^{1}\right), \pi_{r}^{t}\left(x^{2}\right), \cdots, \pi_{r}^{t}\left(x^{s}\right)\right\}$.

(9) Multiply the weight vector $W=\left(w_{1}, w_{2}, \cdots, w_{s}\right)$ by the preference matrix $\Lambda$ in the last round, and then by the Markov state transition matrix $T$, we have

$$
\left[w_{1}, w_{2}, \cdots, w_{s}\right]\left[\begin{array}{cccc}
\pi_{1}^{t}\left(x^{1}\right) & \pi_{1}^{t}\left(x^{2}\right) & \cdots & \pi_{1}^{t}\left(x^{s}\right) \\
\pi_{2}^{t}\left(x^{1}\right) & \pi_{2}^{t}\left(x^{2}\right) & \cdots & \pi_{2}^{t}\left(x^{s}\right) \\
\cdots & \cdots & \cdots & \cdots \\
\pi_{l}^{t}\left(x^{1}\right) & \pi_{l}^{t}\left(x^{2}\right) & \cdots & \pi_{l}^{t}\left(x^{s}\right)
\end{array}\right]\left[\begin{array}{cccc}
T_{11} & T_{12} & \cdots & T_{1 s} \\
T_{21} & T_{22} & \cdots & T_{2 s} \\
\cdots & \cdots & & \cdots \\
T_{s 1} & T_{s 2} & \cdots & T_{s s}
\end{array}\right]=\left[\begin{array}{llll}
x^{1} & x^{2} & \cdots & x^{s}
\end{array}\right.
$$

where $\left[\begin{array}{llll}x^{1} & x^{2} & \cdots & x^{s}\end{array}\right]$ is the preference utility values vector on the scheme set $X$ and $\max \left\{x^{i}\right\}(i=1,2, \cdots, s)$ is the final decision made by the group.

Here we must clarify some points on this group decision-making support approach.

(1) Above procedure goes on entirely and merely based on the interaction between decision-makers and the system without the organization's interruption as in traditional procedure, therefore the decision-making procedure is sped up and this approach can thus meet the requirement of making decisions in limited time.

(2) Each decision maker can defense her opinion, present her explanation, put forward her questions and browse other's opinions anonymously or with her true-name.

(3) The clustering result of the preference values in each round is published and used as a reference for preference adjustment in next round.

(4) Every decision-maker is encouraged to adjust her preference in each round. If every decision-maker insists her initial decision and does not adjust her preference in the group procedure, the group can never achieve consensus and the group decisionmaking makes no sense.

(5) When a decision-maker adjusts her preference, she can make tiny adjustment between two schemes without changing their orders. Such a tiny adjustment represents her accept or reject on the schemes when she cannot have the cake and eat it too, and will be reflected in the state transition matrix.

\section{Conclusion}

This paper researches on the procedure of preference adjustment in group decisionmaking, discusses a description of Markov chain and its main properties, and puts forward the hidden pattern of group preference change based on Markov state transition matrix for group decision-making problems. It also proofs the Markov property of group decision-making procedure and presents the steps for implementing the metasynthesis approach for group decision-making. Further study will firstly focus on the development of such a meta-synthesis GDM approach and the empirical investigation of its effects. 


\section{References}

1. Arrow K J. Social choice and individual values[M ]. New York: JohnWiley\&Sons, 1963

2. Dyer et al. Group Preference Aggregation Rules Based on Strength of Preference, Management Science, 1979, 25 (9): 22 34

3. Keeney et al. Group decision making using cardinal social welfare functions, Management Science, 1975, 22 (4): 430 437

4. French S. Decision Theory: An Introduction to the Mathematics of rationality, Ellis Harwood, Chi Chester, 1986

5. Bordly et al. On the Aggregation of Individual Probability Estimates, Management Science, 1981, 27 (8): 959 964

6. Hochbaum DS and Levin A. Methodologies and algorithms for group-rankings decision, Management Science, 2006, 52 (9): 1394-1408.

7. Fan ZP, Ma J, Jiang YP, Sun YH, and Ma L. A goal programming approach to group decision making based on multiplicative preference relations and fuzzy preference relations. European Journal of Operational Research, 2006, 174 (1): 311-321.

8. Herrera-Viedma E, Martinez L, Mata F, et al. A consensus support system model for group decision-making problems with multigranular linguistic preference relations. IEEE Transactions on Fuzzy Systems, 2005, 13 (5): 644-658.

9. Shen H.Z., Zhao J.D., Wang H.C., et al. Benchmarking service quality from the inside and outside of a service organization by group decision-making, International Conference on Services Systems and Services Management, JUN 13-15, 2005, pp. 502-507

10. Shen H.Z., Hu D.P., Wang H.C., Approaching Consensus Algorithm of Group Decision Based on Clustering Analysis Proceedings of the fifth International Symposium on Knowledge and Systems Sciences, JAIST, Japen, November 10-12, 2004, pp. 267-273. 